\title{
Respuesta al tratamiento oncoespecífico en carcinomas no microcíticos etapa IIIA no quirúrgica
}

\author{
Drialis Díaz Garrido, ${ }^{1}$ Yusbiel J León Valdivies, ${ }^{2}$ Sergio Fernández García, ${ }^{1}$ \\ Manuel Díaz Toledo, ${ }^{3}$ Reynaldo Sánchez de la Osa, ${ }^{4}$ Liliana Osés Herrera ${ }^{5}$
}

\section{Resumen}

El cáncer de pulmón de células no pequeñas incluye los tumores malignos más frecuentes del pulmón. Se realizó un estudio descriptivo, ambispectivo para evaluar la evolución clínica y radiológica en 61 pacientes con cáncer de pulmón de células no pequeñas en estadio IIIA no quirúrgico, tratados con poliquimioterapia y/o radioterapia en el Hospital Benéfico Jurídico en el periodo comprendido entre enero de 2011 y diciembre de 2013. Predominaron los individuos del sexo masculino $(70.5 \%)$ con edad mayor de 60 años (63.9\%). El tratamiento oncoespecífico redujo significativamente la pérdida de peso ( $52.5 \%$ frente a $13.1 \%, p=0.007$ ), el dolor torácico $(55.7 \%$ frente a $27.9 \%, p=0.015)$ y la expectoración (31.1\% frente a $18.0 \%, p=0.043)$. Después del tratamiento oncoespecífico, se encontró un empeoramiento progresivo del estado funcional $(p=0.015)$. Más de la mitad de los sujetos (57.4\%) estaban estables $(27.8 \%$ ) o con alguna respuesta objetiva antitumoral (29.6\%). No obstante, el $42.6 \%$ se hallaba en progresión de la enfermedad. Aunque el tratamiento oncoespecífico no quirúrgico en personas con cáncer de pulmón no microcítico en etapa IIIA produce una mejoría significativa de algunos síntomas, los pacientes empeoran progresivamente su estado funcional, con una baja respuesta objetiva antitumoral.

Palabras clave: Tratamiento oncoespecífico, etapa IIIA, cáncer de pulmón de células no pequeñas.

\begin{abstract}
Summary
Non-small-cell lung cancer includes the most frequent malignant tumors of the lung. A descriptive, ambispective study was carried out to evaluate the clinical and radiological evolution of 61 patients with non-surgical stage IIIA nonsmall-cell lung cancer, treated with polychemotherapy and/ or radiotherapy in the Hospital Benéfico Jurídico in the period between January 2011 and December 2013. Male subjects $(70.5 \%) 60$ years and older $(63.9 \%)$ prevailed. Oncospecific treatment significantly reduced weight loss (52.5\% vs. $13.1 \%, p=0.007)$, thoracic pain $(55.7 \%$ vs. $27.9 \%, p=0.015)$ and expectoration (31.1\% vs. $18.0 \%, p$ $=0.043$ ). After the oncospecific treatment, a progressive worsening of the functional status was found $(p=0.015)$. More than half of the patients $(57.4 \%)$ were stable $(27.8 \%)$ or with some objective antitumor response (29.6\%). Nevertheless, $42.6 \%$ were in progression of the disease. Although non-surgical oncospecific treatment results in a significant improvement of some symptoms in patients with stage IIIA non-small-cell lung cancer, their functional status worsens progressively, with a low objective antitumor response.
\end{abstract}

Key words: Oncospecific treatment, stage IIIA, non-smallcell lung cancer.

\footnotetext{
${ }^{1}$ Especialista en Neumotisiología. Servicio de Neumología. Hospital Benéfico Jurídico. La Habana, Cuba.

2 Especialista en Neumotisiología. Servicio de Medicina Interna. Hospital Gustavo Aldereguía Lima. Cienfuegos, Cuba.

3 Especialista en Neumotisiología. Servicio de Oncología. Hospital Benéfico Jurídico. La Habana, Cuba.

${ }^{4}$ Especialista en Imagenología. Servicio de Imagenología. Hospital Benéfico Jurídico. La Habana, Cuba.

${ }^{5}$ Odontóloga. Residente de segundo año de Estomatología General Integral. Universidad de Ciencias Médicas. Cienfuegos, Cuba.
}

Correspondencia:

Dr. Yusbiel J. León Valdivies

Correo electrónico: ylv87@nauta.cu

Aceptado: 25-07-2016.

Este artículo puede ser consultado en versión completa en http://www.medigraphic.com/actamedica 


\section{INTRODUCCIÓN}

El cáncer de pulmón es una enfermedad del hombre moderno; fue hasta 1842 que se realizó la primera descripción completa de un caso de cáncer de pulmón. ${ }^{1}$ Para 1878 , los tumores de pulmón representaban sólo el 1\% de los cánceres observados durante las autopsias. La cifra se elevó a 10-15\% en la primera parte del siglo XX. ${ }^{2}$ Los cánceres de pulmón de células no pequeñas (CPCNP) son los tumores malignos más frecuentes del pulmón y son la causa más común de ingreso en los servicios de neumología.

En la actualidad, éste es el tipo de cáncer más frecuente en los países desarrollados, representando la primera causa de muerte tanto en hombres como en mujeres; al momento del diagnóstico, más del $40 \%$ de los pacientes tienen enfermedad localmente avanzada. ${ }^{1,2}$

Las neoplasias ocupan en Cuba el primer lugar entre las tasas de defunción global, con 200/100,000 habitantes cada año. Dentro de éstas, los tumores malignos de tráquea, bronquios y pulmón presentan la mayoría de todas las muertes ocurridas por tumores malignos. ${ }^{3}$ Según estadísticas nacionales, en el 2007 enfermaron más de 3,000 personas de uno y otro sexo por este tipo de tumores (una tasa aproximada de 28/100,000 habitantes). El 86\% de las muertes en ese año fueron atribuidas a la exposición al tabaco. ${ }^{4}$

Después del diagnóstico de cáncer de pulmón y la clasificación histológica, la determinación del estadio es un aspecto de gran importancia, con implicaciones terapéuticas y pronósticos. ${ }^{5}$ En el estadio IIIA, donde la enfermedad avanzada es aún locorregional, el individuo puede presentar un tumor abordable quirúrgicamente (tumor seco), donde está indicada la quimioterapia como tratamiento adyuvante y, en ocasiones, se manda radioterapia para controlar la infiltración local.6,7

Los protocolos terapéuticos actuales, que incluyen combinaciones de radioterapia y quimioterapia, suponen una mayor agresividad del tratamiento y, por tanto, la posibilidad de mayores efectos adversos en sujetos con una enfermedad rápidamente debilitante. Esta combinación puede administrarse de forma secuencial, de manera simultánea o alternante. ${ }^{8}$

La conducta estándar en nuestro servicio con las personas que poseen diagnóstico citohistológico de CPCNP en el estadio IIIA son tres opciones: la cirugía en el grupo que tiene criterios iniciales de la misma, la quimioterapia neoadyuvante (tres ciclos y reevaluación posterior) o la quimioterapia (con o sin radioterapia) en quienes en ningún momento de su evolución, desde el diagnóstico o durante la quimioterapia, cumplan con el criterio quirúrgico.

Hasta el momento, aproximadamente entre el 50 y $70 \%$ de los individuos son diagnosticados en estadio avanzado y usualmente incurable (etapas IIIB y IV); sin embargo, la etapa IIIA no tiene cifras despreciables: en nuestro centro (Hospital Neumológico Benéfico Jurídico) representó el 19\% durante el año 2012. Por esta razón, nos motivamos a conocer la evolución clínica y radiológica de estos sujetos luego de recibido el tratamiento oncoespecífico no quirúrgico en el Hospital Neumológico "Benéfico Jurídico" (HNBJ) desde el año 2011 hasta el 2013, por la importancia de conocer la evolución clínica y radiológica en las personas con CPNCP en estadio IIIA no quirúrgico y la utilidad del uso de la quimioterapia y/o radioterapia en esta etapa.

\section{DISEÑO METODOLÓGICO}

Se realizó un estudio descriptivo, ambispectivo para evaluar la evolución clínica y radiológica de los pacientes con cáncer de pulmón de células no pequeñas en estadio IIIA no quirúrgico tratados con poliquimioterapia y/o radioterapia en el Hospital "Benéfico Jurídico" entre enero de 2011 y diciembre de 2013. El universo estuvo constituido por el $100 \%$ de los individuos atendidos con diagnóstico confirmado de cáncer de pulmón de células no pequeñas en estadio IIIA no quirúrgico en el Hospital Benéfico Jurídico en el periodo de estudio. La muestra estuvo constituida por 61 sujetos diagnosticados de cáncer de pulmón de células no pequeñas en estadio IIIA confirmado histológicamente y tratados en nuestro centro con quimioterapia: cisplatino $100 \mathrm{mg} / \mathrm{m}^{2}$ EV el primer día, más etopósido 100-120 mg/m² EV el primer, segundo y tercer días hasta un total de cuatro a seis ciclos, así como radioterapia concurrente; las personas con estado funcional (según escala de Zubrod) entre cero y dos, así como quienes tuvieron estudio tomográfico al diagnóstico y al finalizar el tratamiento. Se excluyeron del estudio los individuos en etapa IIIA con criterio quirúrgico al diagnóstico o durante el tratamiento y los que fallecieron durante el estudio.

La fuente de datos primaria fue el registro del grupo multidisciplinario de atención al paciente oncológico de la institución, así como las estadísticas de cumplimiento de tratamiento del Departamento de Quimioterapia. Se revisaron, además, las historias clínicas de los sujetos que completaron como mínimo cuatro ciclos de tratamiento con citostáticos. Finalmente, se valoraron las tomografías de las personas objeto de estudio realizadas en un equipo multidetector de 64 canales, Somatom Sensation 64 (Siemens Healthcare, Erlangen, Alemania), Ilevando a cabo reconstrucciones a una distancia de $1 \times 0.5 \mathrm{~mm}$ en dirección craneocaudal con una dosis de radiación de $77 \mathrm{mAs}$. Las imágenes fueron interpretadas por el mismo médico especialista.

En cuanto a la recolección de la información, se utilizaron dos métodos: empírico y estadístico. Método empírico: planilla recopiladora de datos, donde se recogieron aspectos demográficos y clínicos: la edad en años; 
género: masculino y femenino, las manifestaciones clínicas al diagnóstico y al final del tratamiento. Los síntomas que se tuvieron en cuenta fueron tos, disnea, hemoptisis, dolor torácico, pérdida de peso, expectoración y disfonía. Para evaluar el estado general del enfermo y determinar el grado de actividad al inicio y final del tratamiento, se empleó la escala de Zubrod. Para calificar la respuesta objetiva antitumoral del paciente se utilizaron los criterios internacionales propuestos por el Comité de Criterios de Evaluación de la Respuesta en Tumores Sólidos, conocido por sus siglas en inglés como RECIST, ${ }^{9}$ que emplea como método imagenológico la tomografía computarizada al diagnóstico y al final del tratamiento. La respuesta se clasificó en: respuesta completa, respuesta parcial, estabilidad y progresión. Método estadístico: se aplicó la prueba de Mc Nemar para el análisis de la evolución de los síntomas al inicio y final del tratamiento, así como el test de independencia chi cuadrado $\left(\chi^{2}\right)$ para el análisis del grado de actividad antes y después del abordaje oncoespecífico no quirúrgico etapa IIIA. En el análisis estadístico también se utilizó el cálculo porcentual. Para el análisis de significación estadística se usó el intervalo de confianza (IC) del 95\% y un nivel de significación $p<0.05$.

Los resultados obtenidos se mostraron en textos, tablas y gráficos (media, desviación estándar, mínimo y máximo), frecuencias acumuladas y porcentuales, estadígrafos de significación y contrastes de hipótesis para muestras relacionadas.

Esta investigación no representó un riesgo para la vida de los participantes porque toda la información fue recopilada de las historias clínicas y plasmada en una planilla de vaciamiento, tratada de forma confidencial y con fines puramente científicos. Se solicitó la autorización de la dirección del hospital, a través del Consejo Científico y la Comisión de Ética Médica. Se asegura la no existencia de conflicto de intereses para el estudio.

\section{RESULTADOS}

Del total de 61 individuos incluidos, $70.5 \%(n=43)$ era del sexo masculino, mientras que el $29.5 \%(n=18)$ restante era del sexo femenino.

Respecto a la distribución de los sujetos según grupos de edad, predominaron aquéllos con edad entre 60 y 69 años $(42.6 \%, n=26)$, seguidos por el grupo entre 40 y 59 años $(36.1 \% \mathrm{n}=22)$. El $19.7 \%$ tenía edad entre 70 y 79 años $(n=12)$, mientras que se encontró sólo una persona con edad mayor de 80 años (1.6\%).

Los síntomas que predominaron antes del tratamiento fueron la tos $(75.4 \%)$, la disnea (63.9\%), el dolor torácico $(55.7 \%)$ y la pérdida de peso $(52.5 \%)$, en ese orden. El síntoma menos frecuente fue la disfonía (6.6\%). Después del tratamiento, los síntomas que obtuvieron una reducción significativa fueron, en orden, la pérdida de peso $(52.5 \%$ versus $13.1 \%, p=0.007)$, el dolor torácico ( $55.7 \%$ versus $27.9 \%, p=0.015)$ y la expectoración (31.1\% versus $18.0 \%$, $\mathrm{p}=0.043)$. La disnea se redujo de manera no significativa en aproximadamente un 20\% (Cuadro 1).

Antes de iniciar el tratamiento, aproximadamente la mitad de los pacientes tenía un estado funcional grado 0 (52.5\%), y un $39.3 \%$ grado 1 . Después de finalizado el tratamiento, si bien no se encontraron diferencias importantes en aquéllos con estado funcional sin restricción de las actividades físicas (52.5\% versus $45.9 \%)$, el porcentaje de individuos con grado I se redujo (39.3\% versus $18.0 \%$ ) y los sujetos con grado 2 $(8.2 \%$ versus $24.6 \%)$ y grado $3(0.0 \%$ versus $11.5 \%)$ aumentaron. Estas diferencias fueron significativas $(p=0.015)$ (Figura 1). Es importante señalar que los síntomas que mejoraron fueron síntomas que tienen poca influencia sobre el estado funcional de los pacientes oncológicos, por lo que durante el estudio, aunque algunos síntomas mejoraron, otros permanecieron en el tiempo, asociados a las reacciones adversas del tratamiento oncológico y la progresión de la enfermedad, lo que puede ser la causa del empeoramiento del estado funcional a pesar de la mejoría de algunos síntomas.

La respuesta completa se alcanzó sólo en dos personas (3.4\%), mientras que el 26.2\% $(n=16)$ logró alcanzar una respuesta parcial y el $27.8 \%(n=17)$ se encontraba en estabilidad. No obstante, el $42.6 \%(n=26)$ de los individuos se halló en progresión de la enfermedad (Figura 2).

\section{DISCUSIÓN}

En nuestro estudio predominaron los sujetos del sexo masculino, con edad mayor de 60 años. Resultados de estudios como el de Moraleja y cols. ${ }^{10}$ evidencian una

\begin{tabular}{|c|c|c|c|c|c|}
\hline \multicolumn{6}{|c|}{$\begin{array}{l}\text { Cuadro 1. Manifestaciones clínicas antes y después } \\
\text { del tratamiento oncoespecífico. }\end{array}$} \\
\hline \multirow{2}{*}{$\begin{array}{l}\text { Manifestaciones } \\
\text { clínicas }\end{array}$} & \multicolumn{2}{|c|}{ Antes } & \multicolumn{2}{|c|}{ Después } & \multirow[b]{2}{*}{$p$} \\
\hline & $\mathrm{n}$ & $\%$ & $\mathrm{n}$ & $\%$ & \\
\hline Tos & 46 & 75.4 & 45 & 73.8 & 0.899 \\
\hline Disnea & 39 & 63.9 & 27 & 44.3 & 0.069 \\
\hline Pérdida de peso & 32 & 52.5 & 8 & 13.1 & $0.007^{\star}$ \\
\hline Dolor torácico & 34 & 55.7 & 17 & 27.9 & $0.015^{\star}$ \\
\hline Hemoptisis & 9 & 14.8 & 8 & 13.1 & 0.823 \\
\hline Expectoración & 19 & 31.1 & 11 & 18.0 & $0.043^{\star}$ \\
\hline Disfonía & 4 & 6.6 & 2 & 3.3 & 0.177 \\
\hline
\end{tabular}


mayor afectación en el grupo etario de 60 a 69 años, con una relación varones/féminas de 2:1. En Estados Unidos, la incidencia de cáncer de pulmón ha pasado de una relación varón/mujer 7:1 hace algo más de una década a una relación $2: 1$, esperándose un incremento exponencial en los próximos años de esta enfermedad en el sexo femenino, en relación con el aumento del hábito de fumar. ${ }^{11}$

En nuestro trabajo, los síntomas más frecuentes de presentación fueron la disnea, la tos, el dolor torácico y la pérdida de peso, en ese orden, observables en más de

Figura 1. Estado funcional antes y después de finalizar el tratamiento.

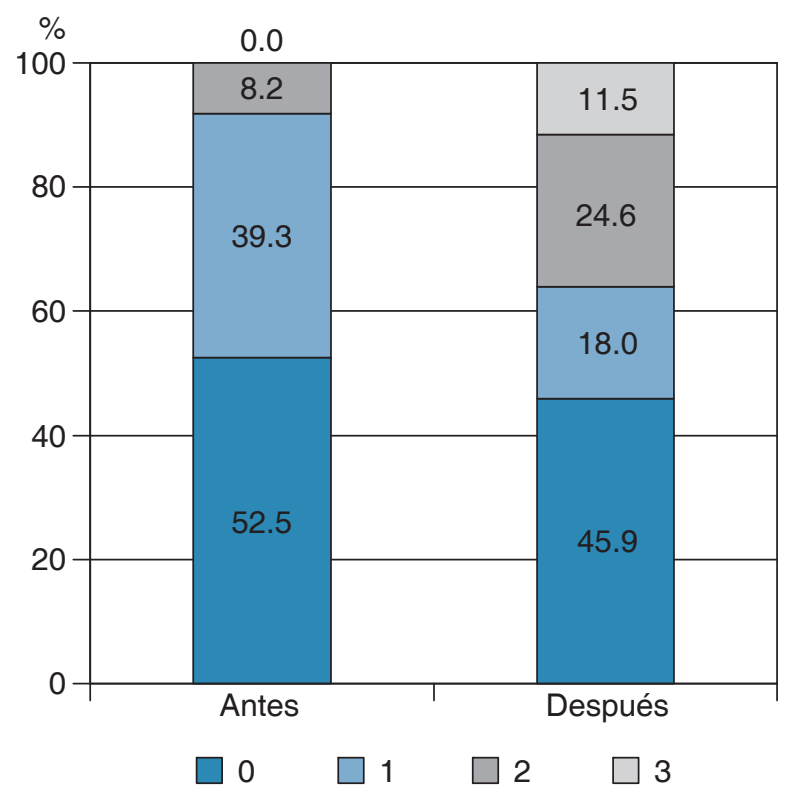

Figura 2. Distribución de los pacientes según la respuesta objetiva antitumoral.

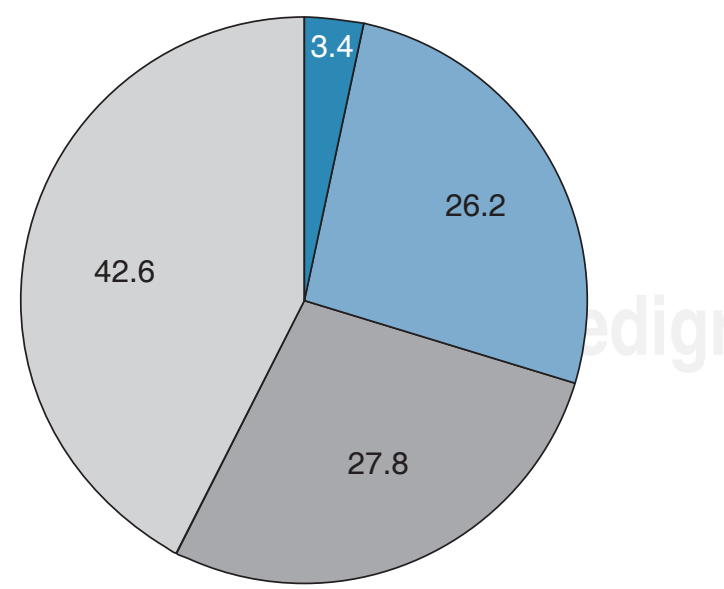

Completa $\square$ Parcial $\square$ Estabilidad $\square$ Progresión la mitad de las personas. La disnea es un síntoma referido por la bibliografía hasta en un $60 \% ; ;^{12,13}$ en nuestro estudio fue de $63.9 \%$, lo cual se puede atribuir a varios factores, como son la adicción tabáquica, que en la mayoría es la explicación del cuadro de insuficiencia respiratoria crónica que padecen estos pacientes.

Se recoge por la literatura que el síntoma inicial más frecuente en el cáncer de pulmón es la tos, ya que está presente en un $70-80 \%$ de los individuos en el momento del diagnóstico. ${ }^{14} \mathrm{~A}$ pesar de ello, es un síntoma muy inespecífico y, por tanto, en muchas ocasiones no es tomado en cuenta por ellos. En nuestra investigación se observó en un $75.4 \%$ de la muestra. Estas cifras están por encima de las obtenidas en un estudio multicéntrico realizado en España en 2003, en el cual la presencia de tos en los sujetos con cáncer de pulmón alcanzó cifras que oscilaron de 15.7\% a $55.8 \% .{ }^{15}$ Muchas veces, el síndrome general caracterizado por astenia, anorexia y pérdida de peso puede ser la carta de presentación en la neoplasia de pulmón, pudiendo aparecer hasta en el $80 \%$ de las personas a lo largo de su enfermedad. Sin embargo, al ser estos síntomas inespecíficos y comunes a disímiles enfermedades los hace poco útiles para un diagnóstico certero de este padecimiento. En la muestra, la pérdida de peso se presentó en el $67.9 \%$, lo cual es un dato alarmante, teniendo en cuenta que en estudios realizados se elevaba al 50\% de pacientes con cáncer de pulmón inoperable y hasta al $78 \%$ en los que estaban en cuidados paliativos. ${ }^{14}$ Las manifestaciones clínicas halladas en la presente investigación concuerdan con otras similares referidas por Montero y su grupo ${ }^{15}$ en España, en un trabajo descriptivo y prospectivo realizado en el área de Coruña. En el presente estudio, el tratamiento oncoespecífico no se relacionó con una mejoría del estado funcional y, si bien más de la mitad de los individuos se encontraban estables o con alguna respuesta objetiva antitumoral, el $42.6 \%$ se hallaba en progresión de la enfermedad. Desde 1972, el tratamiento con radioterapia se consideró un estándar en estos sujetos, con una supervivencia a cinco años menor del 10\%. Un metaanálisis probó que el beneficio de agregar quimioterapia al tratamiento con radioterapia se traduce en una disminución del 10-20\% en el riesgo de muerte. Otro metaanálisis con quimiorradioterapia produjo un beneficio absoluto en la supervivencia global de $5.7 \%$ a tres años y en el control locorregional. ${ }^{16}$

De acuerdo con la más reciente revisión del American Joint Committee on Cancer staging system, el estadio IIIA del CPCNP consiste en $T_{3} N_{1} M_{0}, T_{1-3} N_{2} M_{0}$ y $T_{4} N_{0-1} M_{0}$. En las personas con CPCNP estadio IIIA con situación $\mathrm{N}_{2}$ preoperatoria y/o enfermedad Bulky, se recomienda la radioquimioterapia a base de platino, que puede administrarse simultáneamente en pacientes con buen estado general, aunque se mantiene como tema de debate la incorpora- 
ción o no de la cirugía como tratamiento multimodal. ${ }^{17} \mathrm{En}$ nuestro estudio, se incluyeron individuos en estadio IIIA sin criterio quirúrgico. En un metaanálisis realizado por Sánchez de la Rosa, ${ }^{18}$ se evaluaron los resultados del tratamiento con diferentes esquemas de quimioterapia; de ellos, el más frecuente fue el paclitaxel con cisplatino, que mostró una respuesta objetiva antitumoral que varió entre $21 \%$ y $40.6 \%$. Los hallazgos de nuestro estudio se encuentran en este rango, pues la respuesta objetiva se alcanzó sólo en el 29.6\%.

En el SWOG8805, los sujetos recibieron dos ciclos de cisplatino/etopósido en forma concurrente con radioterapia (45Gy). Las tasas de respuesta fueron del 59\%, y de enfermedad estable del $29 \%{ }^{19}$

En el presente trabajo se describe la respuesta al tratamiento oncoespecífico en la etapa IIIA, evidenciándose mejoría de alguno de los síntomas clínicos y la respuesta objetiva al tratamiento antitumoral, aun cuando el estado funcional empeoró en este grupo de investigación; por lo tanto, hubo respuesta al tratamiento oncoespecífico en la etapa IIIA para las personas incluidas, aun cuando un elevado porciento de ellas presentaron progresión de la enfermedad.

\section{REFERENCIAS}

1. Longo DL, Kasper DL, Jameson JL, Fauci AS, Hauser SL, Loscalzo J. Harrison's Principles of Internal Medicine. [book in CD ROOM]. 18 ed. New York: McGraw-Hill Companies, Inc.; 2012.

2. NCCN. Non-small cell lung cancer. [citado Mar 2012]. Disponible en: http://nccn.com/guidelines/nsclc

3. Siegel R, Ward E, Brawley O, Jemal A. Cancer statistics, 2011: The impact of eliminating socioeconomic and racial disparities on premature cancer deaths. CA Cancer J Clin. 2011; 61: 212236.

4. Rodríguez CA, Martín RA, Hernández CK. Concordancia clínica, tomográfica y anatomopatológica en el cáncer de pulmón. MEDISAN. 2011; 15 (5): 650-656.

5. Amorín Kajatt E. Cáncer de pulmón, una revisión sobre el conocimiento actual, métodos diagnósticos y perspectivas terapéuticas. Rev Peru Med Exp Salud Publica. 2013; 30: 85-92.

6. Rami R. Nueva clasificación TNM del cáncer de pulmón. Arch Bronconeumol. 2009; 45: 159-161.
7. Detterbeck FC, Boffa DJ, Tanoue LT. The new lung cancer staging system. Chest. 2009; 136: 260-271.

8. Vest MT, Tanoue L, Soulos PR, Kim AW, Detterbeck F, Morgemsztern $\mathrm{D}$ et al. Thoroughness of mediastinal staging in stage IIIA non-small cell lung cancer. J Thorac Oncol. 2012; 7: 188-195.

9. Fuente Alonso C de la, Regueiro C, Cajón E, Varcárcel F, García Menocal MI, López-García Gallo C et al. Comparación radio quimioterapia concomitante con Taxol y Vincristina en el carcinoma de pulmón no microcítico localmente avanzado. Arch Bronconeumol. 2008; 40: 1-151.

10. Alonso MR, Sayas CJ, García LR, Coronado PM, Monsó ME, López EA. Utilidad de la tomografía por emisión de positrones en la detección de metástasis ocultas extratorácicas en el carcinoma broncogénico no células pequeñas. Arch Bronconeumol. 2010; 46: 238-243.

11. Schiller JH, Harrington D, Belani CP, Langer C, Sandler A, Krook J et al. Comparison of four chemotherapy regimens for advanced nonsmall-cell lung cancer. N Engl J Med. 2007; 346: 92-98.

12. Paz-Ares LG, Biesma B, Heigener D, von Pawel J, Eisen T, Bennouna J et al. Phase III, randomized, double-blind, placebo-controlled trial of gemcitabine/cisplatin alone or with sorafenib for the first-line treatment of advanced, nonsquamous non-small-cell lung cancer. J Clin Oncol. 2012; 30: 3084-3092.

13. Castro Carpeño J, Belda Iniesta C, Gonzáles Barón M. Carcinoma no microcítico de pulmón: aspectos generales. En: Cortés Funes $\mathrm{H}$, Colomer Bosch R. Tratado de oncología. Segunda edición. Barcelona: Publicaciones Permanyer. 2009.

14. Sánchez De Cos Escuín J. El cáncer de pulmón en España, epidemiología supervivencia y tratamiento actuales. Arch Bronconeumol. 2009; 45: 341-8.

15. Montero C, Rosales M, Otero I, Blanco M, Rodríguez G, Peterga S, y cols. Cáncer de pulmón en el área sanitaria de la Coruña: incidencia, abordaje clínico y supervivencia. Arch Bronconeumol. 2008; 39: 209-16.

16. NICE clinical guideline 121. Lung cancer: the diagnosis and treatment of lung cancer [citado Abr 2011] [42 pantallas]. Disponible en: http:// www.nice.org.uk/ guidance/CG121

17. Edge SB, Byrd DR, Compton CC et al. eds. AJCC Cancer Staging Manual. 7th Ed. Nueva York, NY: Springer; 2010.

18. Bunn P: Translation of the biology of lung cancer to targeted therapies. Educational Book. ASCO 2009. 31 Annual Meeting; 2007, Mayo 12 - 15; San Francisco; 395, 406

19. Ramnath N, Dilling TJ, Harris LJ, Kim AW, Michaud GC, Balekian A, et al. Treatment of Stage III Non-small Cell Lung Cancer: Diagnosis and Management of Lung Cancer, 3rd ed: American College of Chest Physicians Evidence-Based Clinical Practice Guidelines. Chest. 2013; 144: 1743. 